\title{
Elderly Persons Aged 80 Years and Older and Their Nutritional Status
}

\author{
Mona Persenius ${ }^{1}$, Gertrud Glawing ${ }^{2}$, Hans-Bertil Hermansson' ${ }^{2}$, Ingela Karlsson ${ }^{1}$ \\ ${ }^{1}$ Department of Health Sciences, Faculty of Health, Science and Technology, Karlstad University, Karlstad, \\ Sweden \\ ${ }^{2}$ Storfors Municipality, Sweden \\ Email: mona.persenius@kau.se
}

Received 18 February 2014; revised 23 March 2014; accepted 6 April 2014

Copyright (C) 2014 by authors and Scientific Research Publishing Inc.

This work is licensed under the Creative Commons Attribution International License (CC BY). http://creativecommons.org/licenses/by/4.0/

(c) (i) Open Access

\begin{abstract}
A challenge for health care providers is that there will be a distinct rise globally in the number of elderly people aged 80 years and over. Malnutrition is a well-known problem among elderly people; few studies have focused on the nutritional status of those aged over 80, irrespective of whether they live in nursing homes (NHs) or in ordinary housing. The aim of the study was to examine the nutritional status of elderly people aged 80 or older. The study was cross-sectional with 64 elderly persons ( 80 - 100 years old) living in NHs $(n=35)$ or in ordinary housing $(n=29)$. Their nutritional status was assessed with the Mini Nutritional Assessment. Among the elderly people living in NHs, half were at risk and one fourth were suffering from malnutrition. Ten percent of the elderly persons in ordinary housing were found to be at risk; none were malnourished. Thirty-one percent had BMI values $<23$. The majority of the participants ate 2 - 3 meals per day and were prescribed more than three medications daily. Although not statistically significant, well-nourished participants were more likely to be married, and to rate their psychological and physical health very good/good compared to participants who were at risk of becoming malnourished or were malnourished. In conclusion, the results indicate that special attention regarding nutritional intake is required for those aged 80 years and older irrespective of type of accommodation. The RNs have a responsibility to identify the problems and needs and to implement and evaluate nutritional care in this age group. There is a further need for knowledge regarding the effects of both nutritional interventions and of their optimum timing.
\end{abstract}

\section{Keywords}

Elderly, Malnutrition, Mini Nutritional Assessment, Nursing Home, Ordinary Housing 


\section{Introduction}

In nursing homes (NHs), the prevalence of malnutrition is well documented. A multi-national study with 24 pooled studies (4507 people) showed that in NHs in twelve countries from all five continents, half of the residents $>65$ years of age were at risk and $14 \%$ were malnourished [1]. When a modified version of the Nutrition Day project was developed for NHs and used in eight Austrian and 30 German NHs, it was found that 9\% and $17 \%$ respectively out of 2137 residents (age 50 - 106) in 79 units were classified as the malnourished [2]. Overall, malnutrition prevalence rates do not differ significantly between elderly people aged $>65$ years in NHs in Netherlands (27\%) and Germany (26\%) [3]. In Sweden, the prevalence of malnutrition in NHs was recently found to be $30 \%$ [4].

Among older people $>65$ years and living in ordinary housing, the prevalence of malnutrition varies from $6 \%$ [1] to 14\% [5]. In Sweden, 15.7\% (60 - 96 years of age) are malnourished [6]. These variations in prevalence can be explained by the use of different definitions of malnutrition, the setting of the study, the use of different research methods, various underlying disease, and variation in the ages of the elderly people included. The focus of this study is under nutrition but the terms "malnutrition" and "under nutrition" are used interchangeably in accordance with the literature.

In most populations around the globe, people are living longer and the number of people living beyond the age of 80 is increasing [7] [8], and the trend is that more and more elderly people are living at home [9]. These figures are related not only to a healthier elderly population, as these older people are living at their own home regardless of whether they are healthy or not or in need of home care service [9], but also to a weakening of municipal finances leading to a halted expansion or cutbacks of the care. Between 2000 and 2005, 18,000 beds were closed in NHs while only half of the municipalities expanded their home help service at the same time [10]. The sharp cutback in beds combined with the insufficient expansion of home care services has increased the number of elderly people queuing for admission to NHs. However, during 2013, the maximum waiting time for $\mathrm{NH}$ admission decreased compared with the previous year according to the Swedish Association of Local Authorities and Regions [11]. The waiting time now varies between 0 and 156 days within the municipalities [11].

There is a body of evidence regarding the prevalence of malnutrition and risk of malnutrition among the elderly population aged $>50$ years living in NHs, but there is little research on the nutritional status of the elderly aged $>80$ years, irrespective of whether they are living in NHs or at home. By examining the nutritional status of older people, preventive efforts can be initiated where the need exists. Therefore, the aim of the study was to examine the nutritional status of elderly people aged 80 or older.

\section{Methods}

A descriptive cross-sectional study design was conducted [12], comparing elderly persons living in NHs with elderly persons living in ordinary housing, using a nutritional assessment instrument.

\subsection{Setting}

The study was conducted in three NHs and among persons living in ordinary housing, all within one municipality with about 5000 inhabitants. The NHs provide nursing care around the clock for persons with extensive need for this care and all meals are available from a central kitchen.

\subsection{Participants}

For persons aged 80 or older living in NHs, the inclusion criteria was: Swedish-speaking residents. Excluded in the study were older persons receiving enteral and/or parenteral nutrition and those in the end-stage of life. Forty-five out of 55 persons fulfilled these criteria but eight declined participation and a further two persons were excluded due to them being unable to answer the questions, leaving 35 included persons.

Persons aged 80 or older living in ordinary housing were identified via the population register, providing about 200 potential participants. The participants were ranked according to age and a strategic random sample of the first person was accomplished by lottery. From this person, every fourth person up and down in age was included $(\mathrm{n}=50)$. However, 18 persons declined participation and three persons had unknown addresses, leaving 29 included persons. The inclusion criteria were: Swedish-speaking with cognitive ability to answer the Mini Nutritional Assessment (MNA) and questionnaires and managing without home help services. All 29 persons 
fulfilled the inclusion criteria. A total of 64 elderly persons were finally included in the study.

\subsection{Data Collection}

The data collection was carried out by using an assessment instrument with five additional questions.

\subsubsection{Instrument}

The MNA developed by Guigoz et al. [13] was used for this study. MNA includes a total of 18 questions to assess the nutritional status of older persons. The instrument consists of two parts; an initial assessment (part 1) and a final assessment (part 2). The instrument's first part consists of six items that assess the person's food intake, weight loss, mobility, psychological stress or acute disease, neuropsychological problems, and body mass index (BMI). The responses are measured by a scale of $0-3$ points, where 0 or 1 indicates lack of functions and 2 or 3 indicates normal functions. Summation of the scores is made and the maximum score is 14 . A result of eleven points or less denotes that a risk of malnutrition exists and the second part of the MNA is performed. Here, all participants had the full MNA assessment conducted.

The second part includes twelve questions on housing, drug intake, presence of wounds, the ability to feed themselves, food and water intake, independent assessment of health and nutritional status, and measurement of mid-arm (MAC) and calf circumference (CC). The responses are measured by a scale ranging from 0 to 2 points. The responses are summarized and added to the summation from part one. The maximum score in total for the first and second part is 30. If the total score is between 17 and 23.5 points, the person is judged to be at risk of malnutrition; at a score below 17 points, there is malnutrition [13]. The instrument's credibility is supported by its development and validation in both healthy and sick elderly populations [13] [14] and it has been used in several settings and across populations [15]. Registered nurses (RNs) and enrolled nurses (ENs) have made similar estimates when using the first part of the MNA, the Short Form-Mini Nutritional Assessment (MNA-SF), proving its reliability [16].

In addition, three questions related to demographics were added: marital status (married/cohabitant or single), type of living (urban or rural) and type of housing (villa, apartment, $\mathrm{NH}$, or block of serviced flats). A further two questions about perceived psychological and physical health (each classified as very good, good, neither good nor bad, bad, very bad, or cannot answer the question) were also added.

\subsubsection{Procedure}

Older people living in NHs received oral and written information by dietary agents, that is, ENs with special education in and experience of nutritional assessments. After two weeks, the dietary agents renewed their contact with the participants and if participation in the study was accepted an appointment was scheduled and written and informed consent was obtained. Where the respondent was judged by the dietary agents as not having the cognitive ability to give informed consent and answer the questionnaire regarding demographic facts, a relative was consulted. Copies of the MNAs from the patients' medical records were obtained from the RN. In the studied municipality it was routine at the time of the study to make an assessment with the MNA for all residents.

Written information about the study was posted to older persons living at home. Two weeks later they were contacted via telephone by one of the researchers $(\mathrm{H}-\mathrm{BH})$ and, if participation was accepted, a time for an appointment was scheduled. A nutritional assessment with the MNA was carried out by one of the authors (GG) or by the dietary agent together with the respondent. At the same time, written, informed consent was obtained. This took place between May and June 2011.

\subsection{Data Analysis}

The computer program IBM Statistical Package for Social Sciences (SPSS) version 20.0 was used for analyzing the data, with descriptive statistics displaying the frequencies, percentages, mean values and standard deviations regarding MNA, demographics and perceived health. Pearson's Chi-square tests were used to compare the differences between nutritional status and gender, marital status, type of living, and perceived psychological and physical health. In the results, the classifications "villa" and "apartment" were merged to create one, "ordinary housing", and "risk of under nutrition" and "under nutrition" were combined together into "malnutrition/at risk". Regarding psychological and physical health, the response alternatives "very good" and "good" were merged 
into one group and "bad" and "very bad" were merged into another group. Responses classified as "cannot answer the question" were not included in the analysis.

\subsection{Ethical Consideration}

The study was approved by an ethical research committee, Dnr 2010/290, and was conducted according to the Helsinki declaration [17] and the Ethical Guidelines for Nursing Research in the Nordic Countries [18]. The heads of departments for each NH gave consent for the study. The Patient Data Act [19] was adhered to for the acquisition of each MNA copy, and the handover of information was listed in each nursing journal of the RN in charge.

All participants took part voluntarily in the study after giving their oral and written informed consent. Respondents could withdraw their participation at any time without explanation. Their data were treated confidentially. The questionnaire and the MNA were coded to enable interconnection of the documents without compromising anonymity.

\section{Results}

The mean age of the total number of respondents was 86.9 years (ranging from 80 to 100). In NHs the mean age was 88.8 years. For persons living in ordinary housing, the mean age was 84.6 years. There were more women than men included in the study and two-thirds were single. The majority lived in urban areas (Table 1).

Table 1. Overview over demographic data.

\begin{tabular}{|c|c|c|c|c|c|}
\hline & Mean & SD & Range & $\mathrm{n}$ & $(\%)$ \\
\hline \multicolumn{6}{|l|}{ Age } \\
\hline Total & 86.9 & 4.6 & $80-100$ & & \\
\hline Nursing homes & 88.8 & 4.6 & $80-100$ & & \\
\hline Ordinary living & 84.6 & 3.5 & $80-93$ & & \\
\hline \multicolumn{6}{|l|}{ Gender } \\
\hline Male & & & & 28 & 43.8 \\
\hline Female & & & & 36 & 56.3 \\
\hline \multicolumn{6}{|l|}{ Marital status } \\
\hline Married/cohabitant & & & & 22 & 34.4 \\
\hline Single & & & & 42 & 65.6 \\
\hline \multicolumn{6}{|l|}{ Living } \\
\hline Urban & & & & 53 & 82.8 \\
\hline Rural & & & & 11 & 17.2 \\
\hline \multicolumn{6}{|l|}{ Ordinary housing } \\
\hline Villa & & & & 24 & 37.5 \\
\hline Apartment & & & & 5 & 7.8 \\
\hline \multicolumn{6}{|l|}{ Nursing homes } \\
\hline Nursing homes & & & & 17 & 26.6 \\
\hline Serviced flats & & & & 18 & 28.1 \\
\hline
\end{tabular}




\subsection{Nutritional Condition}

In total, half of the respondents were judged to be well-nourished (53.1\%, $n=34 / 64)$ according to the MNA, while almost one-third were at risk of being malnourished $(32.8 \%, n=21)$ or were malnourished $(14 \%, n=9)$. Among the elderly people living in NHs, about half of the respondents were at risk $(51.4 \%, n=18 / 35)$ and one-fourth were malnourished $(25.7 \%, \mathrm{n}=9)$, while among the elderly living in ordinary housing, only a few were at risk $(10.3 \%, n=3 / 29)$ and none were malnourished.

The result of the MNA screening (part one) is shown in Table 2. In total, the majority reported no loss of appetite, good mobility, did not suffer from stress or acute disease and had a BMI $\geq 23$. One-fifth reported weight loss, mainly those from the NHs.

The results of part two of the MNA screening are shown in Table 3. In total, the majority reported that they take more than three medications daily, ate three meals per day, could eat on their own, had MAC greater than $22 \mathrm{~cm}$ and CC of $31 \mathrm{~cm}$ or greater. Twelve percent reported pressure sores or skin ulcers. Eleven percent could eat by themselves but with difficulties or required feeding assistance, had MAC $\leq 22$ and $17.2 \%$ had CC of less than $31 \mathrm{~cm}$, all within the NHs.

Table 2. The MNA screening part 1 in relation to type of housing.

\section{MNA screening part 1}

A. Declined food intake over the past three months due to loss of appetite, digestive problems, chewing or swallowing difficulties

Severely poor

Moderately poor

No loss of appetite

B. Weight loss during the last three months

Weight loss $>3 \mathrm{~kg}$

Does not know

Weight loss 1 - $3 \mathrm{~kg}$

No weight loss

C. Mobility

Bed or wheelchair bound

Can get out of the bed/chair but cannot walk

Can move around with or without support

D. Have suffered psychological stress or acute disease

yes

no

\section{E. Neuropsychological problem}

Severe dementia or depression

Mild dementia

No psychological problem

F. Body Mass Index (BMI)

BMI less than 19

BMI 19 to less than 21

BMI 21 to less than 23

BMI 23 or more

G. Can live independently

no

yes
Total $n=64(\%)$

Nursing home $n=35$

Ordinary housing $\mathrm{n}=29$ 
Table 3. The MNA screening part 2 in relation to type of living.

\section{MNA screening part 2}

H. Take $>3$ prescription drugs daily

yes

no

I. Pressure sores or skin ulcers

yes

no

J. Full meals consumed daily

One meal

Two meals

Three meals

K. Consumption of protein-rich foods every day (3 choices yes/no)

0 - 1 yes

2 yes

3 yes

L. Consuming $>2$ serving of fruits and vegetables per day

no

yes

M. Cups of fluid/water consumed/day

$<3$ cups

3 - 5 cups

$>5$ cups

\section{N. Mode of feeding}

Requires feeding assistance

Self-fed with some difficulty

Self-fed without any problem

O. Self-rated nutritional status

Has major malnutrition

Does not know or has moderate malnutrition

No nutritional problems

P. Self-rated health compared with others in same age

Not as good

Does not know

As good

Better

Q. Mid Arm Circumference (MAC)

MAC less than $21 \mathrm{~cm}$

MAC 21 to $22 \mathrm{~cm}$

MAC greater than $22 \mathrm{~cm}$

R. Calf Circumference (CC)

CC less than $31 \mathrm{~cm}$

CC $31 \mathrm{~cm}$ or greater
Total

$\mathrm{n}=64(\%)$

Nursing home $n=35$

Ordinary housing $n=29$

46(71.9)

28

18

18(28.1)

7

11

8(12.5)

6

2

56(87.5)

29

\section{2}

47(73.4)

4

\section{2}

24(37.5)

38(59.4) 


\subsection{Perceived Health}

When the participants compared their self-rated general health condition with others in the same age, 56.3\% answered that their health condition was the same or better than others in the same age, and $43.8 \%$ felt worse or did not know (Table 3). In total, 75.9\% $(n=44 / 58)$ perceived their psychological health as very good/good, $17.2 \%(n=10)$ perceived it as neither good nor bad, while $6.9 \%(n=4)$ perceived it as bad/very bad. Their physical health was perceived as very good/good by $66.7 \%(n=40 / 60)$, as neither good nor bad by $20 \%(n=12)$, and as bad/very bad by $13.3 \%(n=8)$.

\subsection{Comparisons between Participants with Different Nutritional Conditions}

There were statistically significantly more well-nourished participants (76.5\%) in ordinary living compared to participants who were at risk of becoming malnourished or were malnourished $(10 \%, \mathrm{p}<0.001)$. In regard to gender, there was no statistically significant difference. Although not statistically significant, there were tendencies in the results that more well-nourished participants were married, and that they rated their psychological and physical health as very good/good compared to participants who were at risk of becoming malnourished or were malnourished (Table 4).

\section{Discussion}

\subsection{Study Result}

The results showed that in NHs half of the respondents were at risk and one-fourth were malnourished according to the MNA, findings that are in line with other studies [1] [2] [20]. Living in NHs might mean a fivefold risk of malnutrition compared with living in the municipality [21], but a recent study indicates an improvement in these figures, as 30\% of the $\mathrm{NH}$ residents in Sweden with a mean age of 86.3 are suffering from malnutrition, compared with historical data from 1996 when $71 \%$ were malnourished [4].

Table 4. Gender, marital status, type of living and health in relation to nutritional condition.

\begin{tabular}{|c|c|c|c|}
\hline & Well-nourished $n=34(\%)$ & Malnourished/at risk n = 30 (\%) & $p$-value \\
\hline Gender & & & 0.283 \\
\hline Male & $17(50)$ & 11(36.7) & \\
\hline Female & $17(50)$ & 19(63.3) & \\
\hline Marital status & & & 0.081 \\
\hline Married & 15(44.1) & $7(23.3)$ & \\
\hline Non-cohabitant & 19(55.9) & 23(76.7) & \\
\hline Type of living & & & $<0.001$ \\
\hline Ordinary housing & $26(76.5)$ & $3(10)$ & \\
\hline Nursing home & $8(23.5)$ & $27(90)$ & \\
\hline Psychological health & & & 0.093 \\
\hline Very good/good & $27(84.4)$ & $17(65.4)$ & \\
\hline Neither good nor bad/bad/very bad & $5(15.6)$ & $9(34.6)$ & \\
\hline Cannot answer & 2 & 4 & \\
\hline Physical health & & & 0.099 \\
\hline Very good/good & 25(75.7) & $15(55.6)$ & \\
\hline Neither good nor bad/bad/very bad & $8(24.3)$ & $12(44.4)$ & \\
\hline Cannot answer & 1 & 3 & \\
\hline
\end{tabular}


In this study, only ten percent of the elderly persons in ordinary housing were found to be at risk; none were malnourished. This result is less than was reported in the study of Johansson et al. [22] where 14.5\% were found to be malnourished. Our findings also contradict other findings [23] that revealed no differences between elderly persons living in NHs (51 - 97 years old) and elderly persons living in ordinary housing (58 - 86 years old) when measured with MNA. However, being 80 years old and older carries a significant and almost eightfold greater risk of becoming malnourished compared with those aged between 65 - 69 years according to Lopez-Jornet et al. [21].

Although the elderly people ate two to three full meals a day at the NHs, the majority were malnourished or at risk of becoming malnourished. One reason for this could be that the RNs often overestimate the elderly people's actual food intake [24] [25]. Several studies have shown that elderly people in NHs eat less than half of their food portion [26] [27], a factor associated with malnutrition [26]. Furthermore, long overnight fasts could also be a crucial component [28]-[30]. In addition, it is known that when living in NHs, elderly people have little influence on the food served and the timing of meals [31]. The municipal nutritional care is insufficient and it is for example the responsibility of the nursing manager to provide routines that decrease the time of overnight fasts and increase the elderly persons involvement in making decisions about their nutrition.

The results showed that the majority of participants were prescribed more than three medications. Adverse side-effects, for example dryness in the mouth, constipation and nausea, may negatively affect the patients' food intake and it is well known that well-nourished elderly people take fewer medications when compared with those who are malnourished [32] [33]. A potentially inappropriate prescription of medication for elderly people living in special housing is reported [34] [35] and polypharmacy, defined as the prescription of five drugs or more at once, is increasing among the elderly population, resulting in higher morbidity and mortality [34] [36]. The RNs have a key role in medication management, such as monitoring and evaluating the drug treatment in discussions with the responsible physician, but this role is rarely discussed and depending on how the RNs define their role this might have consequences on the safety and quality of the care [37].

Pressure sores or skin ulcers were found in $12 \%$ of the participants. Low nutritional intake, low BMI and undesired weight loss are all significantly related to pressure ulcers [38]. A lack of systematic risk assessments as well as evidence-based intervention has been identified among RNs in NHs [39]. Improvements in risk assessment tools [39], a more complete assessment when patients are at risk or where established malnutrition is found, followed by individually tailor-made nutritional support, are recommended [38]. Such interventions are also of importance in supporting the care of elderly persons living at home.

Although the results showed that the majority of participants perceived their psychological and physical health as very good/good, $34 \%$ to $44 \%$, respectively, among the malnourished participants perceived their health as low or neutral. Lower self-perceived health is a known risk factor for malnutrition among home-living elderly people [22] and those elderly people living in NHs [28]. The elderly people's self-perceived low well-being might be influenced by a combination of malnutrition and long overnight fasts ( $>11 \mathrm{hrs)} \mathrm{[40].}$

\subsection{Methodological Considerations}

A strength of this study was the use of MNA as it is a well validated and reliable instrument, developed for a geriatric population like the one in this study. Furthermore, the dietary agents were familiar with performing nutritional assessments and using the MNA as it was routine in the studied municipality. The fact that there are no differences found between RNs' and ENs' nutritional assessments regarding the MNA-short form (SF) [15] is a further strengthening of the reliability of the instrument. A less skewed gender distribution with a larger male sample might have had an impact on the results.

\section{Conclusions, and Implications for Nursing and Future Research}

In conclusion, the results of the present study indicate that special attention regarding nutritional intake is required for those aged 80 years and older irrespective of type of housing. The RNs have a responsibility to identify the problems and needs of the elderly people and to implement and evaluate their nutritional support and care. There is a need for more knowledge regarding the effects of not only nutritional interventions but also of the optimum timing of these by further evaluation research. Further studies with larger sample sizes are needed in order to confirm the results of this study. Also, similar studies including elderly persons receiving a home help service would be of great value. 


\section{Acknowledgements}

The authors would like to thank the dietary agents for help with the data collection.

\section{References}

[1] Kaiser, M., Bauer, J., Rämsch, C., Uther, W., Guigoz, Y., Cederholm, T., et al. (2010) Frequency of Malnutrition in Older Adults: A Multinational Perspective Using the Mini Nutritional Assessment. The American Geriatrics Society, 58, 1734-1748. http://dx.doi.org/10.1111/j.1532-5415.2010.03016.x

[2] Valentini, L., Schindler, K., Schlaffer, R., Bucher, H., Mouhieddine, M., Steinger, K., et al. (2009) The First Nutrition Day in Nursing Homes: Participation May Improve Malnutrition Awareness. Clinical Nutrition, 28, 109-116. http://dx.doi.org/10.1016/j.clnu.2009.01.021

[3] Van Nie-Visser, N.C., Meijers, J.M.M., Schols, M.G.A., Lohrmann, C., Bartholomeyczik, S. and Halfens, R.J.G. (2011) Comparing Quality of Nutritional Care in Dutch and German Nursing Homes. Journal of Clinical Nursing, 20, 25012508. http://dx.doi.org/10.1111/j.1365-2702.2011.03761.x

[4] Törmä, J., Winbladh, U., Cederholm, T. and Saletti, A. (2013) Does Undernutrition Still Prevail among Nursing Home Residents? Clinical Nutrition, 32, 562-568. http://dx.doi.org/10.1016/j.clnu.2012.10.007

[5] Stratton, R.J. and Elia, M. (2011) Prevalence of Malnutrition. In: Sobotka, L., Ed., Basics in Clinical Nutrition, 4th Edition, Galen, Prague, 46-52.

[6] Fagerström, C., Palmqvist, R., Carlsson, J. and Hellström, Y. (2011) Malnutrition and Cognitive Impairment among People 60 Years of Age and above Living in Regular Housing and Special Housing in Sweden: A Population-Based Cohort Study. International Journal of Nursing Studies, 48, 863-871. http://dx.doi.org/10.1016/j.ijnurstu.2011.01.007

[7] Statistiska Centralbyrån (2012) Demografiska rapporter-sveriges framtida befolkning 2012-2060. Statistiska Centralbyrån, Stockholm. (In Swedish)

[8] United Nations. World Population Ageing: 1950-2050. http://www.un.org/esa/population/publications/worldageing19502050/

[9] Socialstyrelsen (2013) Äldrevård och omsorg den 1 oktober 2012. (In Swedish) http://www.socialstyrelsen.se/publikationer2013/2013-4-15

[10] The National Board of Health and Welfare (2007) Current Developments in Care of the Elderly in Sweden. http://www.socialstyrelsen.se/Lists/Artikelkatalog/Attachments/9296/2007-131-40_200713140.pdf

[11] Sverigeskommuner och landsting (Swedish Association of Local Authorities and Regions) (2013) Öppna jämförelser 2013. Vård och omsorg om äldre. Jämförelser mellan kommuner och län. (In Swedish) http://www.skl.se/

[12] Polit, D.F. and Tatano, B.C. (2012) Nursing Research: Generating and Assessing Evidence for Nursing Practice. 9th Edition, Lippincott Williams and Wilkins, Philadelphia.

[13] Guigoz, Y., Vellas, B. and Garry, P.J. (1994) Mini Nutritional Assessment: A practical Assessment Tool for Grading the Nutritional State of Elderly Patients. In: Vellas, B., Ed., The Mini Nutritional Assessment (MNA), Supplement No 2, Serdi Publisher, Paris, 15-59.

[14] Vellas, B., Guigoz, Y. and Garry, P.J. (1999) The Mini Nutritional Assessment (MNA) and Its Use in Grading the Nutritional State of Elderly Patients. Nutrition, 15, 116-122. http://dx.doi.org/10.1016/S0899-9007(98)00171-3

[15] Guigoz, Y. (2006) The Mini Nutritional Assessment (MNA) Review of the Literature-What Does It Tell Us? Journal of Nutrition, Health and Aging, 10, 466-487.

[16] Bååth, C., Hall-Lord, M.L., Idvall, E., Wiberg-Hedman, K. and Larsson, B.W. (2008) Interrater Reliability Using Modified Norton Scale, Pressure Ulcer Card, Short Form-Mini Nutritional Assessment by Registered and Enrolled Nurses in Clinical Practice. Journal of Clinical Nursing, 17, 618-626. http://dx.doi.org/10.1111/j.1365-2702.2007.02131.x

[17] World Medical Association (2008) Declaration of Helsinki-Ethical Principles for Medical Research Involving Human Subjects. http://www.wma.net/en/30publications/10policies/b3/

[18] Northern Nurses Federation (2003) Ethical Guidelines for Nursing Research in the Nordic Countries. Vård i Norden: Nordic Journal of Nursing Research, 23, 1-19.

[19] Patientdatalag (2008) http://www.notisum.se/rnp/sls/lag/20080355.htm

[20] Verbrugghe, M., Beeckman, D., Van Hecke, A., Vanderwee, K., Van Herck, K., Clays, E., et al. (2013) Malnutrition and Associated Factors in Nursing Home Residents: A Cross-Sectional, Multi-Centre Study. Clinical Nutrition, 32, 438-443. http://dx.doi.org/10.1016/j.clnu.2012.09.008

[21] Lopez-Jornet, P., Saura-Perez, M. and Llevat-Espinosa, N. (2013) Effect of Oral Health Dental State and Risk of Mal- 
nutrition in Elderly People. Geriatrics Gerontology International, 13, 43-49.

http://dx.doi.org/10.1111/j.1447-0594.2012.00853.X

[22] Johansson, Y., Bacharach-Lindström, M., Carstensen, J. and Ek, A.-C. (2008) Malnutrition in a Home-Living Older Population: Prevalence, Incidence and Risk Factors. A Prospective Study. Journal of Clinical Nursing, 18, 1354-1364. http://dx.doi.org/10.1111/j.1365-2702.2008.02552.x

[23] Saava, M. and Kisper-Hint, I.-R. (2002) Nutritional Assessment of Elderly People Living in Nursing House and at Home in Tallin. The Journal of Nutrition, Health \& Aging, 1, 93-95.

[24] Simmons, S.F. and Reuben, D. (2000) Nutritional Intake Monitoring for Nursing Home Residents: A Comparison of Staff Documentation, Direct Observation, and Photography Methods. Journal of American Geriatric Society, 48, 209213.

[25] Suominen, M., Laine, T., Routasalo, P., Pitkala, K.H. and Rasanen, L. (2004) Nutrient Content of Served Food, Nutrient Intake and Nutritional Status of Residents with Dementia in a Finnish Nursing Home. The Journal of Nutrition, Health \& Aging, 8, 234-238.

[26] Suominen, M., Muurinen, S., Routasalo, P., Soini, H., Suur-Iski, I., Peiponen, A., et al. (2005) Malnutrition and Associated Factors among Aged Residents in All Nursing Homes in Helsinki. European Journal of Clinical Nutrition, 59, 578-583. http://dx.doi.org/10.1038/sj.ejcn.1602111

[27] Xia, C. and McCutcheon, H. (2006) Mealtimes in Hospital—Who Does What? Journal of Clinical Nursing, 15, 12211227. http://dx.doi.org/10.1111/j.1365-2702.2006.01425.x

[28] Ödlund, O.A., Koochek, A., Ljungqvist, O. and Cederholm, T. (2005) Nutritional Status, Well-Being and Functional Ability in Frail Elderly Service Flat Residents. European Journal of Clinical Nutrition, 59, 263-270. http://dx.doi.org/10.1038/sj.ejcn.1602067

[29] Dahl Eide, H., Aukner, C. and Iversen, P.O. (2012) Nutritional Status and Duration of Overnight Fast among Elderly Residents in Municipal Nursing Homes in Oslo. Vård i Norden: Nordic Journal of Nursing Research, 105, 20-24.

[30] Söderström, L., Adolfsson, T., Rosenblad, A., Frid, H. and Saletti, A. (2013) Mealtime Habits and Meal Provision Are Associated with Malnutrition among Elderly Patients Admitted to Hospital. Clinical Nutrition, 32, 281-288. http://dx.doi.org/10.1016/j.clnu.2012.07.013

[31] Sidenvall, B., Fjellström, C. and Ek, A.C. (1996) Cultural Perspectives of Meals Expressed by Patients in Geriatric Care. International Journal of Nursing Studies, 33, 212-222. http://dx.doi.org/10.1016/0020-7489(95)00042-9

[32] Chen, C.C., Bai, Y.Y., Huang, G.H. and Tang, S.T. (2007) Revisiting the Concept of Malnutrition in Older People. Journal of Clinical Nursing, 16, 2015-2026. http://dx.doi.org/10.1111/j.1365-2702.2006.01867.x

[33] Chang, C.C. and Roberts, B.L. (2011) Malnutrition and Feeding Difficulty in Taiwanese Older with Dementia. Journal of Clinical Nursing, 20, 2153-2161. http://dx.doi.org/10.1111/j.1365-2702.2010.03686.x

[34] Liu, G.G. and Christensen, D.B. (2002) The Continuing Challenge of Inappropriate Prescribing in the Elderly: An Update of the Evidence. Journal of the American Pharmacist Association (Wash), 42, 847-857. http://dx.doi.org/10.1331/108658002762063682

[35] Milos, V., Rekman, E., Bondesson, Å., Eriksson, T., Jakobsson, U., Westerlund., T., et al. (2013) Improving the Quality of Pharmacotherapy in Elderly Primary Care Patients through Medication Reviews: A Randomized Controlled Study. Drugs Aging, 30, 235-246. http://dx.doi.org/10.1007/s40266-013-0057-0

[36] Hajjar, E.R., Cafiero, A.C. and Hanlon, J.T. (2007) Polypharmacy in Elderly Patients. American Journal of Geriatric Pharmacotherapy, 5, 345-351. http://dx.doi.org/10.1016/j.amjopharm.2007.12.002

[37] Nordin Olsson, I., Wätterbjörk, I. and Blomberg, K. (2014) Registered Nurses’ Perception of Their Professional Role Regarding Medication Management in Nursing Care of the Elderly. Journal of Nursing Education and Practice, 4, 153-161.

[38] Shahin, E.S.M., Meijers, J.M.M., Scols, J.M.G.A., Tannen, A., Halfens, R.J.G. and Dassen, T. (2010) The Relationship between Malnutrition Parameters and Pressure Ulcers in Hospitals and Nursing Homes. Nutrition, 26, 886-889. http://dx.doi.org/10.1016/j.nut.2010.01.016

[39] Fossum, M., Alexander, G.L., Göransson, K.E., Ehnfors, M. and Ehrenberg, A. (2011) Registered Nurses’ Thinking Strategies on Malnutrition and Pressure Ulcers in Nursing Homes: A Scenario-Based Think-Aloud Study. Journal of Clinical Nursing, 20, 2425-2435. http://dx.doi.org/10.1111/j.1365-2702.2010.03578.x

[40] Ebrahimi, Z. and Wijk, H. (2009) En hypotesgenererande studie av nattfastans längd, undernäring och hälsorelaterad livskvalitet hos äldre. Vård i Norden: Nordic Journal of Nursing Research, 91, 45-48. (in Swedish) 